\title{
Safe sedation for the non-anaesthetist $\mathrm{\Sigma}^{\mathrm{ew}}=$
}

\author{
Authors: Hakeem Yusuff, ${ }^{A}$ Amit Prakash $^{B}$ and Stephen Webb ${ }^{C}$
}

Sedation has become an essential part of many invasive medical procedures. However, over the years, there have been concerns about the safety of sedation techniques. Various combinations of drug-based and non-drug-based approaches are used for procedural sedation depending on patient factors and the anticipated discomfort associated with each procedure. The common denominator for successful practice for a sedationist is knowledge, adequate training and a mechanism for revalidation.

KEYWORDS: Sedation, monitoring, capnography, training

\section{Introduction}

The term 'conscious sedation' has been used to describe the level of sedation that can be safely administered in settings outside the operating theatre by appropriately trained individuals. It describes a technique in which the use of a drug or drugs produces a state of depression of the central nervous system that enables treatment to be carried out, but during which verbal contact with the patient is maintained throughout the period of sedation. The drugs and techniques used to provide conscious sedation should carry a margin of safety wide enough to render loss of consciousness unlikely. ${ }^{1}$ This review summarises the key points presented at the RCP's medical conference titled 'Safe sedation for the non-anaesthetist' on 4 June 2015.

\section{Pharmacology (Dr Joe Arrowsmith)}

Sedative drugs are usually administered orally, intravenously or via inhalation. If using the intravenous or inhalational routes, the drugs used can and must be titrated to effect (see below). It is preferable to use single drugs because they are easier to titrate to effect and safer than concurrent administration of two or more drugs. Drugs in combination typically produce synergistic effects, and differ in times to onset and peak effect, and hence can be unpredictable or difficult to titrate to effect. Safety margins can be narrowed, increasing the likelihood

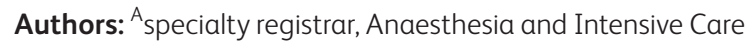
Medicine, Health Education North West, Manchester, UK;

${ }^{B}$ consultant anaesthetist, Addenbrookes Hospital, Cambridge, UK; C ${ }^{C}$ consultant cardiothoracic anaesthesia and intensive care medicine, Papworth Hospital, Cambridge, UK of overdose, loss of consciousness, respiratory depression and the need for airway interventions. ${ }^{1}$ However, for painful procedures, it is often necessary to combine a sedative agent with an analgesic agent in the form of either an intravenous opiate or local anaesthesia. Here we describe the traditional and newer agents used for sedation.

Midazolam is the most widely used benzodiazepine for procedural sedation. It can be used on its own or in combination with an opioid (eg fentanyl). It has a shorter halflife than the other benzodiazepines $(2-3 \mathrm{~h})$; the peak effect occurs 12-15 min after administration and it has no active metabolites. An initial bolus of $1-3 \mathrm{mg}$ is given intravenously initially and titrated to effect with $0.5 \mathrm{mg}$ boluses to a maximum of $5 \mathrm{mg}$.

Fentanyl is a synthetic opiate that is 100 times more potent than morphine. It has a rapid onset and offset (usually approximately $30 \mathrm{~min}$ ) when administered in small doses $(0.25-0.5 \mu \mathrm{g} / \mathrm{kg})$. Fentanyl is often used in combination with midazolam, with which it acts synergistically to produce sedation and analgesia. It is important to give the fentanyl first before titrating midazolam to effect.

Propofol is a phenolic derivative that can provide a range of sedation right up to general anaesthesia, depending on the dose. It is best administered as an infusion to produce steady-state plasma concentrations. Propofol has a quick onset and offset and progression from conscious sedation to deep sedation can occur very quickly, especially if boluses are given. There is evidence that use of propofol to provide sedation for long cardiac interventions by appropriately trained practitioners is safe, effective and practical. ${ }^{2}$

Dexmedetomidine is an $\alpha-2$ adrenoceptor agonist with analgesic, sympatholytic and anxiolytic effects. It is licenced in the UK for sedation in intensive-care units only, but has been used successfully for procedural sedation in children. ${ }^{3}$

\section{Patient selection (Dr Libby Haxby)}

Inadequate pre-assessment has been a recurring factor in sedation-related adverse events and poor outcomes for all specialties. The elderly, morbidly obese and patients with multiple comorbidities can present with challenges, which might suggest the need to plan for the procedure to be done in theatre and for sedation to be provided by an anaesthetist. Similarly, patients with potential difficult airways as measured by the Mallampati scoring system can be identified, which would be helpful in assessing the practitioners' ability to manage any airway or respiratory emergencies that might arise. 
Table 1. ASA grading.

\begin{tabular}{lll}
$\begin{array}{l}\text { ASA classification } \\
\text { ASA I }\end{array}$ & $\begin{array}{l}\text { Patient characteristics } \\
\text { A normal healthy patient }\end{array}$ & $\begin{array}{l}\text { Examples } \\
\text { Healthy; no smoking, no or very minimal drinking }\end{array}$ \\
ASA II & $\begin{array}{l}\text { A patient with mild systemic disease } \\
\text { Smoker; more than minimal drinking; pregnancy; obesity; well controlled } \\
\text { diabetes; well controlled hypertension; mild lung disease }\end{array}$ \\
& $\begin{array}{l}\text { A patient with severe systemic disease. } \\
\text { Not incapacitating }\end{array}$ & $\begin{array}{l}\text { Diabetes; poorly controlled hypertension; distant history of MI, CVA, } \\
\text { TIA, cardiac stent; COPD, ESRD with dialysis; active hepatitis; implanted } \\
\text { pacemaker; ejection fraction < } 40 \% \text {; congenital metabolicabnormalities }\end{array}$ \\
ASA IV & $\begin{array}{l}\text { A patient with severe systemic disease } \\
\text { that is a constant threat to life }\end{array}$ & $\begin{array}{l}\text { Recent history of MI, CVA, TIA, cardiac stent; on-going cardiac ischaemia } \\
\text { or valvular abnormality; implanted ICD, ejection fraction <28 }\end{array}$ \\
ASA V & $\begin{array}{l}\text { A moribund patient who is not } \\
\text { expected to survive without the } \\
\text { operation }\end{array}$ & $\begin{array}{l}\text { Ruptured abdominal or thoracic aortic aneurysm; intra-cranial bleed with } \\
\text { mass effect; ischaemic bowel with significant cardiac pathology. }\end{array}$ \\
& $\begin{array}{l}\text { A patient who has already been } \\
\text { declared brain dead and whose organs } \\
\text { ASA VI }\end{array}$ & \\
& are being retrieved for transplant & \\
\hline
\end{tabular}

The addition of ' $E$ ' indicates emergency surgery. ASA = American Society of Anesthesiologists; COPD = chronic obstructive pulmonary disorder; $C V A=$ cerebrovascular accident; ESRD = end-stage renal disease; $\mathrm{ICD}$ = implantable cardioverter defibrillator; $\mathrm{MI}$ = myocardial infarction; TIA = transient ischaemic attack.

It is important for sedation practitioners to decide at this stage if they would be able to ventilate the patient or not. ${ }^{1}$ The pre-operative interview also provides an opportunity to review the patient's previous experience with sedation, explain the procedure and potential risks and obtain consent. The American Society of Anesthesiologists' (ASA) classification has been used to classify patients presenting for procedural sedation (Table 1). ${ }^{4}$ Patients who are ASA I or II are deemed fit for conscious sedation, whereas patients who are ASA III-V must be assessed and discussed with a consultant anaesthetist.

\section{Monitoring and equipment (Dr David Whittaker)}

Pulse oximetry, non-invasive blood-pressure monitoring and end-tidal $\mathrm{CO}_{2}$ monitoring are the minimum monitoring standard required for conscious sedation. ${ }^{1,5}$ Electrocardiographic monitoring should be added in complex procedures, in patients with underlying heart disease and in patients with multiple comorbidities. Translating from anaesthetic practice, there is strong evidence for the addition of end-tidal $\mathrm{CO}_{2}$ monitoring (Fig 1) for detection of early apnoea detection and indirect monitoring of cardiac ouput. ${ }^{6}$ The information provided is used in combination with periodic clinical assessments to ensure the patient is appropriately sedated with cardiovascular and respiratory function maintained. The bispectral index monitor has the potential to contribute to reduction in sedative doses and early detection of respiratory depression and deeper levels of sedation. ${ }^{7}$ A recent review of all studies so far was inconclusive and emphasised the need for more studies. ${ }^{8}$

Furthermore, emergency equipment - including resuscitation equipment, a continuous high-flow oxygen delivery system, intubation equipment and drugs - should be available. As far as possible, procedural sedation should be undertaken on a tipping trolley to facilitate emergency management of potential aspiration and hypotension.

\section{Recovery and discharge (Dr Liz Williams)}

There have been several reports of patients developing cardiorespiratory compromise on the ward after procedural sedation. Initial assessment of sedation practice in 2001 by the Academy of Medical Royal Colleges confirmed that excessive use of flumazenil and poor or a lack of appropriate recovery facilities contributed to sedation-related adverse events. It is important that patients receive exactly the same level of monitoring in the recovery phase as they did during the procedure, including end-tidal $\mathrm{CO}_{2}$. Emergency equipment, drugs and high-flow oxygen should be available. Staff involved in the recovery of sedated patients should have the same level of training as operating theatre recovery nurses. The Aldrete scoring system was initially designed to guide recovery nurses in identifying patients who are fit for discharge for ward-based care after general anaesthesia. ${ }^{9}$ This system has been adapted for patients who have undergone conscious or deep sedation, but does not take into account pain control and postoperative nausea and vomiting.

\section{Training (Dr Kevin Haire)}

All sedation practitioners should complete structured training in the safe practice of sedation and basic life-support skills. For conscious sedation, the National Institute for Health and Care Excellence's guidelines ${ }^{10}$ recommend that at least one member of the team performing the procedure requiring sedation must have intermediate life support skills. Simulation has been used as a tool to facilitate training in sedation and management of common clinical emergencies, including human factors. ${ }^{11}$ Medical specialties that have sedation as a core competency have developed specialty-specific accredited training, which is assessed at the annual review of competency progression. Trained sedation practitioners should undergo regular review and appraisals and aim to undertake 12 hours of continuingprofessional development every 5 years. 

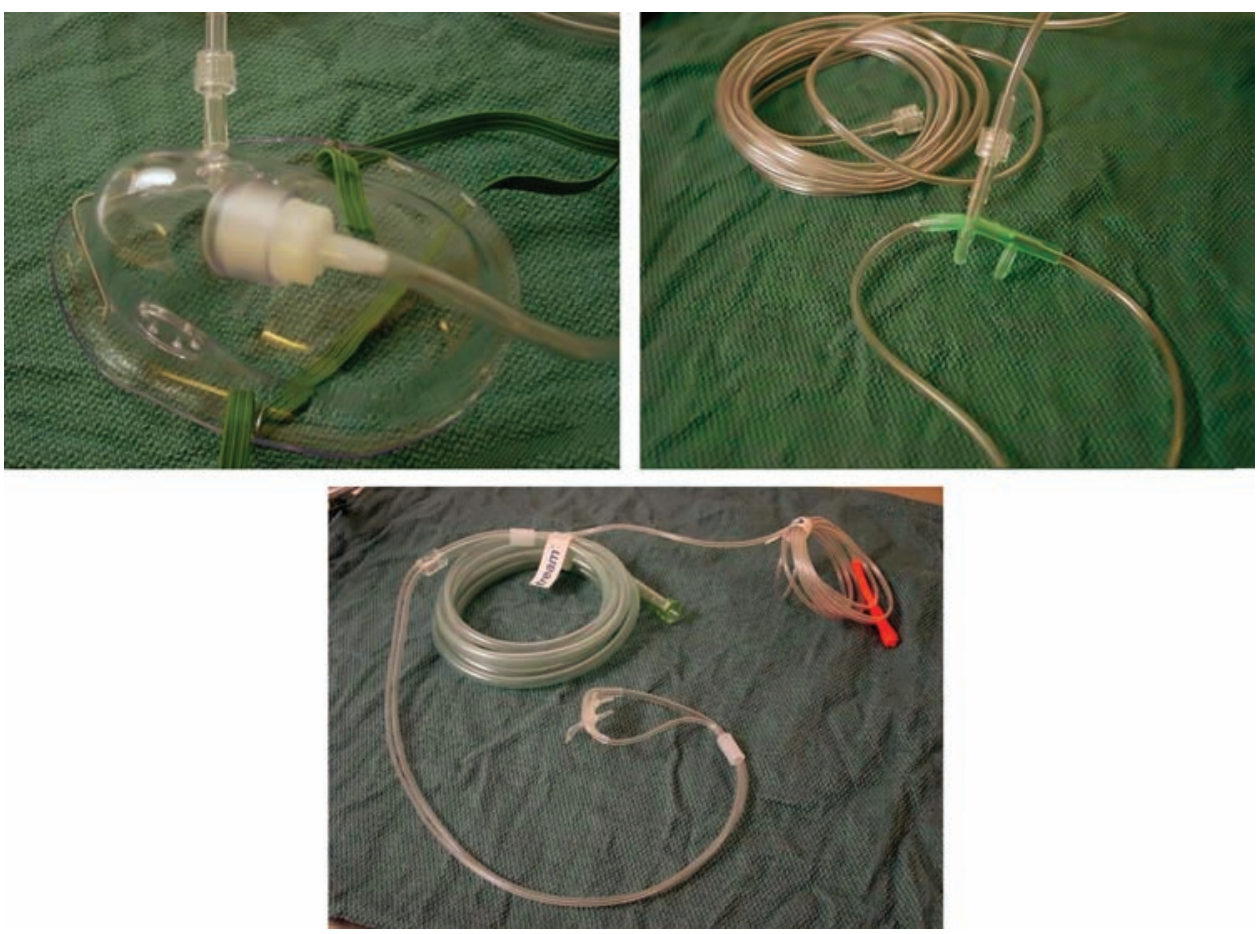

Fig 1. Oxygen delivery devices with end-tidal $\mathrm{CO}_{2}$ monitoring. Reproduced with permission from www.capnography.com.

\section{Conclusion}

Sedation-related adverse events have been in the limelight lately (eg Michael Jackson, Joan Rivers) and investigations have highlighted several factors that suggest an attitude of complacency. In response to this, there has been a focus on the need to standardise sedation practice, which has increased the profile of sedation practice among both patients and healthcare staff. Particular attention to training of personnel, pre-sedation assessment, monitoring and recovery has led to a drop in the incidence of adverse events. Additionally clinical governance processes, including clinical audit and incident reporting, have helped to ensure that opportunities for improvements are identified.

\section{References}

1 UK Academy of Medical Royal Colleges. Implementing and ensuring safe sedation practice for healthcare procedures in adults. London: Academy of Medical Royal Colleges, 2013.

2 Salukhe TV, Willems S, Derewitz I et al. Propofol sedation administered by cardiologists without assisted ventilation for long cardiac interventions. Europace 2012;14:325-30.

3 McMorrow SP, Abramo TJ. Dexmedetomidine sedation: uses in pediatric procedural sedation outside the operating room. Pediatr Emerg Care 2012;28:292-6.

4 American Society of Anesthesiologists. ASA Physical Status Classification System. Schaumburg, IL: ASA, 2014. Available online at www.asahq.org/resources/clinical-information/asa- physical-status-classification-system [Accessed 29 February 2016].

5 Association of Anaesthetists of Great Britain \& Ireland. AAGBI safety statement. The use of capnography outside the operating theatre. London: AAGBI, 2011.

6 Soto RG, Fu ES, Vila H, Miguel RV. Capnography accurately detects apnoea during monitored anaesthesia care. Anaeth Analg 2004;99:379-82.

7 Miner JR, Biros MH, Seigel T, Ross K. The utility of the bispectral index in procedural sedation with propofol in the emergency department. Acad Emerg Med 2005;12:190-6.

8 Williams A, Ruddock Y. The use of bispectral index monitoring (BIS) in conscious sedation. Emerg Med J 2015;32:414-5.

9 Aldrete JA, Kroulik D. A postanesthetic recovery score. Anesth Analg 1970;49:924-34.

10 National Institute for Health and Care Excellence. Sedation in children and young children. London: NICE, 2010.

11 Boet S, Bould D, Fung L et al. Transfer of learning and patient outcome in simulated crisis resource management: a systematic review. Can J Anaesthesia 2014;61:571-82.

12 Aldrete JA. The post anesthesia recovery score revisited. J Clin Anesth 1995;7:89-91.

Address for correspondence: Dr S Webb, Papworth Hospital NHS Foundation Trust, Papworth Everard, Cambridge CB23 3RE, UK

Email: stephen.webb@nhs.net 